\title{
Organoaxial Rotation of Stomach Delineated on MDCT
}

\author{
Rahul Dev ${ }^{1}$ \\ ${ }^{1}$ Assistant professor, Department of Radiology and Imaging, All India Institute of Medical Sciences (AlIMS), Rishikesh, \\ Uttarakhand, India
}

Corresponding author: Dr. Rahul Dev, Flat No-5/1, Type IIB Flats, AlIMS Rishikesh Residential Complex, Rishikesh, Uttarakhand-249203, India.

DOI: $10.21276 / \mathrm{ijcmsr} .2018 .3 .2 .14$

How to cite this article: Rahul Dev. Organoaxial rotation of stomach delineated on MDCT. International Journal of Contemporary Medicine Surgery and Radiology. 2018;3(2):B58-B59.

\section{A B S T R A C T}

Introduction: Gastric volvulus poses as operative emergency in vast majority of scenario with albeit nonspecific clinical symptoms. Imaging plays a definite role in diagnosis as well as detection of any complications.

Case report: An old age patient presented with nonspecific symptoms raising suspicion of gastric volvulus with imaging findings confirming rather organoaxial rotation of stomach with associated sliding hiatal hernia.

Conclusion: Timely and accurate diagnosis is needed for gastric ischemia and perforation can be prevented in vast majority.

Keywords: Stomach Volvulus, Computed Tomography, Rotation, Sliding Hiatal Hernia

\section{INTRODUCTION}

Volvulus of the stomach poses as a surgical emergency and a challenging radiological diagnosis. The associated clinical symptoms are nonspecific in the majority of cases, including nausea, vomiting and epigastric pain. Because the condition is rarely diagnosed clinically henceforth radiological investigations have a major role to play more so to avoid life threatening complications of ischemia and infarction. ${ }^{1}$ Computed tomography (CT) has largely replaced conventional contrast studies in the evaluation of gastric torsion and volvulus, which often pose challenges for clinicians due to the non-specific symptoms. Gastric volvulus is rare, but ominous condition where the stomach rotates along its long or short axis $180^{\circ}$, leading to a closed loop type of obstruction. In those patients where less than $180^{\circ}$ of stomach rotation is present it will be wise to call it organoaxial or mesenteroaxial rotation rather than volvulus as there are no associated obstructive features. ${ }^{2}$ Organoaxial volvulus accounts for approximately two-thirds of cases of gastric volvulus as per morphological classification with the mesenteroaxial encompassing rest of one third cases. Patients with acute gastric volvulus typically present with epigastric pain, nausea, and vomiting whereas those with chronic symptoms present with nonspecific symptoms such as abdominal pain, early satiety, dyspnoea or dysphagia. The chronic form of gastric volvulus is more common than the acute form. In terms of clinical scenario the acuity and severity of symptoms associated with volvulus direct the management.

\section{CASE REPORT}

A 70 year old female patient presented to the outpatient clinic with intermittent symptoms of dull epigastric pain associated with early satiety and occasional vomiting for last two months duration. There was no associated hematemesis. Clinically the bowel sounds were present and abdomen

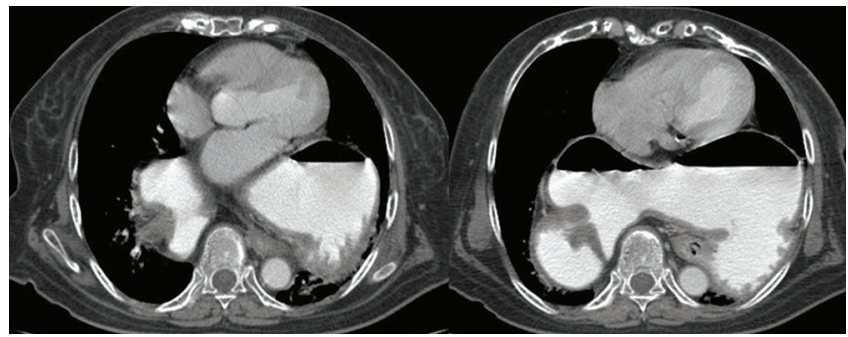

Figure-1: Axial contrast enhanced CT images of upper abdomen show oral contrast distended stomach showing air fluid level being higher up in position, causing focal indentation over left atrium secondary to diaphragmatic eventration. The stomach is also seen to be rotated along its long axis with greater curvature facing anteriorly and lesser curvature, fundus and antropyloric region facing posteriorly depicting organoaxial rotation.

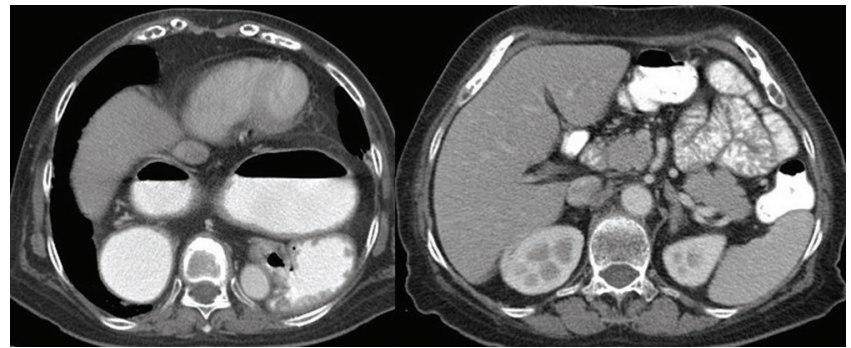

Figure-2: Axial contrast enhanced CT images at a slightly caudal level shows a sliding hiatal hernia with gastroesophageal junction seen anterolateral to descending aorta. Furthermore there is presence of oral contrast within visualised small and large bowel loops excluding complete obstruction. 
was soft and non-distended on palpation. Rest of clinical examination and laboratory parameters were within normal limits. The patient underwent contrast enhanced CT scan of abdomen as part of imaging work up which revealed contrast filled non dilated stomach. The stomach was seen to be higher up in position with secondary eventration of posterior part of diaphragm with in turn stomach indenting cardiac chambers particularly left atrium. The stomach was seen rotated in such configuration along its long axis so that the greater curvature is seen facing anteriorly and lesser curvature including antrum and fundus being posteriorly placed (Fig 1). There was associated sliding hiatal hernia with low lying gastroesophageal junction. There was smooth passage of contrast in small bowel loops distally (Fig 2).

\section{DISCUSSION}

The stomach is an uncommon site for volvulus. The peak incidence occurs in the fifth decade of life. Patients with acute gastric volvulus typically present with upper abdomen pain, nausea and vomiting and is a surgical emergency requiring prompt diagnosis and treatment. In organoaxial volvulus the stomach rotates along its long axis with the greater curvature facing upwards and the lesser curvature facing caudally in the abdomen with fundus and antrum being rotated posteroinferiorly and anterosuperiorly respectively. ${ }^{1}$

If the volvulus is complete with more than greater than $180^{\circ}$ rotation it leads to obstructed dilated fluid filled stomach showing retained positive oral contrast, wall thickening and adjacent fluid or fat stranding. If positive oral contrast material is administered, it is retained in the stomach. However, there are cases with less than $180^{\circ}$ rotation leading to partial obstruction depicting distal passage of ingested contrast material through the stomach into the duodenum. Patients with associated paraoesophageal hiatal hernia are at risk for developing secondary organoaxial rotation of the stomach. Other predisposing factors include phrenic nerve palsy, eventration and post traumatic hernia. This group of patients usually lack clinical signs and symptoms of obstruction with no imaging findings suggestive of obstruction. In such cases, it is more accurate to describe the stomach as having an organoaxial position rather than organoaxial volvulus, although it can lead to frank volvulus in future as complication. ${ }^{2}$ The similar findings are seen in the present case. Mesenteroaxial volvulus is a less common type of volvulus, which occurs when the stomach rotates along its short axis, with the resultant position of antrum above the level of the gastroesophageal junction.

Multi detector $\mathrm{CT}$ often is performed when the diagnosis is in question and helps confirm the rotation of the stomach, including rotation axis, level and degree of obstruction and associated hiatal hernia if any. Differential diagnoses based on imaging features include hiatus hernia, postoperative changes following oesophagectomy with gastric pull through and lower oesophageal diverticulum. ${ }^{3}$ Once the diagnosis is confirmed gastric decompression should be attempted and surgical treatment is undertaken with aim of immediate reduction of volvulus and prevention of recurrence in future. $^{4}$

\section{CONCLUSION}

If timely accurate diagnosis is made and prompt surgical repair is performed gastric ischemia and perforation can be prevented in vast majority averting further complications and mortality.

\section{REFERENCES}

1. Peterson CM, Anderson JS, Hara AK, Carenza JW, Menias CO. Volvulus of the gastrointestinal tract: appearances at multimodality imaging. Radiographics 2009;29 (5):1281-93.

2. Guniganti $\mathrm{P}$, Bradenham $\mathrm{CH}$, Raptis $\mathrm{C}$, Menias CO, Mellnick VM. CT of Gastric Emergencies. Radiographics. 2015;35(7):1909-21.

3. Twists and turns in the body: an imaging spectrum. Luk SY, Fung KH. Hong Kong Med J. 2010;16(5):390-6.

4. Khan RA, Chana RS, Wahab S HK. A perplexing clinical picture in a patient of acute gastric volvulus: a case report. J Paediatr (new series) 2007;12:212-4.

Source of Support: Nil; Conflict of Interest: None

Submitted: 18-03-2018; Accepted: 21-04-2018; Published online: 10-05-2018 